\title{
Theoretical Research on Ellipsoidal Structure Methane Gas Detection Based on Near Infrared Light Sources of PbSe Quantum Dots
}

\author{
Xiaoxue Xing, ${ }^{1}$ Yanmin Lei, ${ }^{1}$ Weiwei Shang, ${ }^{1,2}$ Limin Du, ${ }^{1}$ and Pan Guo ${ }^{3}$ \\ ${ }^{1}$ College of Information Engineering, Changchun University, Changchun 130012, China \\ ${ }^{2}$ College of Automotive Engineering, Jilin University, Changchun 130000, China \\ ${ }^{3}$ School of Science and Technology, College of Humanities \& Sciences, Northeast Normal University, Changchun 130000, China
}

Correspondence should be addressed to Xiaoxue Xing; xiaoxue8184@126.com

Received 15 March 2016; Accepted 15 January 2017; Published 12 February 2017

Academic Editor: Sergio Bietti

Copyright (C) 2017 Xiaoxue Xing et al. This is an open access article distributed under the Creative Commons Attribution License, which permits unrestricted use, distribution, and reproduction in any medium, provided the original work is properly cited.

\begin{abstract}
To improve the precision and sensitivity of the detection in near infrared gas detection system, the selection of light source and design of gas chamber structure are two key links. In this paper, the near infrared (NIR) light sources fabricated with PbSe quantum dots (QDs) and a new gas cell structure using an ellipsoid reflector were designed to test the concentration of methane $\left(\mathrm{CH}_{4}\right)$. The double wavelengths differential detection method was used in the paper. The signal wavelength is $1.665 \mu \mathrm{m}$ from the NIR QD-based light source with $5.1 \mathrm{~nm}$ PbSe QDs. The reference wavelength is $1.943 \mu \mathrm{m}$ from the NIR QD-based light source with $6.1 \mathrm{~nm} \mathrm{PbSe}$ QDs. The experimental results show that the differential gain signal could be enhanced 80 times when the major axis, the focus, and the open length of the ellipsoid reflector are $4.18 \mathrm{~cm}, 3.98 \mathrm{~cm}$, and $0.36 \mathrm{~cm}$, respectively. The structure will be convenient for the signal amplifying, $\mathrm{AD}$ converting, and other process in the latter circuits, and therefore both the detection sensitivity and precision can be improved.
\end{abstract}

\section{Introduction}

Methane $\left(\mathrm{CH}_{4}\right)$ is a flammable and explosive gas. In recent years, great injuries and property losses were caused by frequent gas explosions, which has increasingly attracted the attention of the general public and the government. So, it has become an urgent problem to detect the concentration of methane immediately and accurately. In addition, methane is also a major greenhouse gas in the atmosphere, whose concentration needs to be detected exactly [1-4].

Existing methods for methane detection rely mostly on vector catalytic combustion, thermal conductivity, optical interferometry, and infrared absorption. Among various available methods, infrared spectroscopy absorption has its unique advantages over the other techniques $[5,6]$. The sensing parts of infrared spectroscopy absorption gas detection system include an infrared launch system, a gas chamber, and a photoelectric conversion device. The selection of light source and design of gas chamber structure are two key links to improve the accuracy and sensitivity of detection. Recently, the emergence of the quantum cascade laser provides an alternate solution as infrared light source. However, quantum cascade laser as light source is expensive with complicated designs and structures, making it less desirable for the rising needs on portable, simple, and low-cost infrared gas sensors.

Semiconductor quantum dots (QDs) have been investigated and have shown very unique properties, such as the quantum confined optical property [7-13]. They usually possess high photoluminescence (PL) quantum yield (QY) with size dependent tunable wavelength emissions, which makes them promising for light conversion [14-16]. Among them, the PbSe bulk material has a small bandgap of $0.28 \mathrm{eV}$ at room temperature and a very large exciton Bohr radius of $46 \mathrm{~nm}$ [17-24]. As a result, PbSe QDs show very strong quantum confinement and high quantum yield in NIR region. Their band edge photoluminescence peaks span over a wide infrared wavelength region of 1-4 $\mu \mathrm{m}$ [25-29]. The wavelength can be adjusted merely by changing the particle size to 
cover the particular absorption frequencies of many kinds of gases in the NIR region. As a matter of fact, the NIR emitting QDs with narrow bandgaps have great application potential in gas detection. Compared to other infrared light sources for gas detection including infrared thermal emitter and semiconductor lasers, these NIR QDs have relative high modulate rate without the large thermal inertia, narrow emitting band without obvious interference, low cost, and small volume.

The normal gas cell in the infrared gas detection system can be divided into two categories: transmission type gas cell and reflecting type gas cell. When the narrowband light source is used to measure $\mathrm{CH}_{4}$ concentration, the transmission type gas cell is more prone to produce coherent noise, which will affect the sensitivity of the system; the reflecting type gas chamber is more prone to produce interference noise, which can also bring a certain impact on the sensitivity of system [30-32].

In this paper, the NIR PbSe QDs and a new gas cell structure using an ellipsoid reflector were designed to analyze the concentration of $\mathrm{CH}_{4}$. Based on the double wavelengths differential detection method, the NIR PbSe QDs with the particle sizes of 5.1 and $6.1 \mathrm{~nm}$ are chosen as the NIR light sources. Due to the narrow PL spectra of NIR PbSe QDs, the filter needed in the conditional detection system can be omitted. Considering the low sensitivity of traditional gas cell structures, a new gas cell structure using an ellipsoid reflector is designed. The math model of the ellipsoid reflector is proposed. And the relationship between the parameters of the ellipsoid reflector and the light intensity of the detector is described. It can be proved that the structure can enhance the intensity of the output signal.

\section{Establishment and Analysis of the Detection Model}

In order to improve the sensitivity of infrared detection, a new gas cell structure using an ellipsoid reflector is designed, as shown in Figure 1. First of all, we establish the theoretical math model. $a$ and $b$ are the semimajor axis and the semiminor axis of the ellipsoid reflector, respectively. As shown in Figure $1, c=\sqrt{a^{2}-b^{2}}$ and $e=c / a<1$ and the focal point coordinates are $F_{1}(+c, 0)$ and $F_{2}(-c, 0)$. The signal light source $A$ is placed at $F_{1}$; the reference light source $B$ is placed at a defocus position, and the detector is on $F_{2}$.

The NIR QDs light source can be treated as a cosine radiation. Radiation intensity in any direction $\theta$ is

$$
I(\theta)=\sum_{i} I_{0}\left(\lambda_{i}\right) \cos \theta
$$

where $I_{0}\left(\lambda_{i}\right)$ is radiation intensity of wavelength $\lambda_{i}$ along axes.

According to Beer-Lambert law [33-35], the relationship between the reflected light radiation intensity and the incident light radiation intensity can be expressed as

$$
\begin{aligned}
I_{t}(\theta) & =\sum_{i} I_{0}\left(\lambda_{i}\right) \cos \theta \rho\left(\lambda_{i}\right) e^{-\sigma\left(\lambda_{i}\right) C L} \\
& =\sum_{i} I_{0}\left(\lambda_{i}\right) \cos \theta \rho\left(\lambda_{i}\right) e^{-\alpha\left(\lambda_{i}\right) L},
\end{aligned}
$$

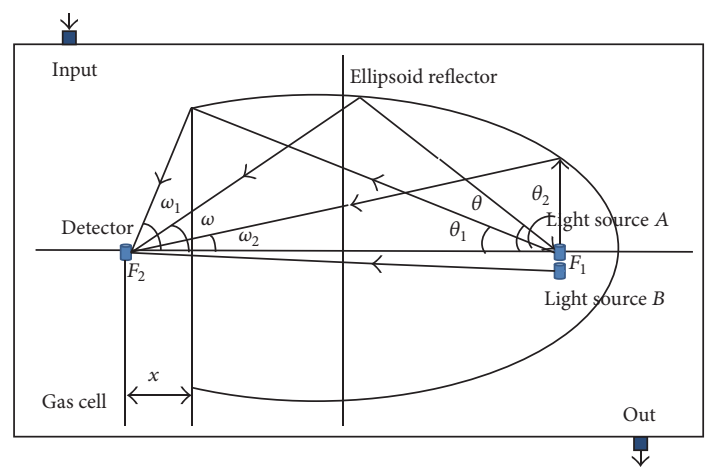

Figure 1: Diagram of ellipsoid reflector gas cell.

where $\sigma\left(\lambda_{i}\right)$ is absorption cross section of $\mathrm{CH}_{4}$; $\mathrm{C}$ is the concentration of $\mathrm{CH}_{4} ; \rho\left(\lambda_{i}\right)$ is ellipsoid reflectivity; $L$ is the length of absorption path; $\alpha\left(\lambda_{i}\right)$ is absorption coefficient of $\mathrm{CH}_{4}$.

The radiant flux received by detector can be written as

$$
\begin{aligned}
& \Phi^{\prime} \\
& =\sum_{i} I_{0}\left(\lambda_{i}\right) e^{-\alpha\left(\lambda_{i}\right) L_{0}} \frac{S}{L_{0}^{2}} \\
& \quad+\int_{0}^{2 \pi} d \varphi \int_{\theta_{1}}^{\theta_{2}} \sum_{i} I_{0}\left(\lambda_{i}\right) \rho\left(\lambda_{i}\right) e^{-\alpha\left(\lambda_{i}\right) L} \sin \theta \cos \theta d \theta,
\end{aligned}
$$

where $L_{0}$ is the distance between two focuses and $S$ is the reception area of detector. The transmission distance $L$ is a fixed value for the radiations of different angles.

The receiving model of detector can be written as

$$
R(\omega)=R_{0} \cos ^{4} \omega,
$$

where $R_{0}$ is the response extent of detector. When $\lambda_{A}$ and $\lambda_{B}$ are adjacent, $R_{A 0}=R_{B 0}$.

The output electric signal converted by the detector is

$$
\begin{aligned}
V^{\prime}= & R_{0} \sum_{i} I_{0}\left(\lambda_{i}\right) e^{-\alpha\left(\lambda_{i}\right) L_{0}} \frac{S}{L_{0}^{2}} \\
+ & 2 \pi \int_{\theta_{1}}^{\theta_{2}} \sum_{i} I_{0}\left(\lambda_{i}\right) \rho\left(\lambda_{i}\right) e^{-\alpha\left(\lambda_{i}\right) L} \sin \theta \cos \theta d \theta \\
& \cdot \int_{\omega_{1}}^{\omega_{2}} R(\omega) d \omega .
\end{aligned}
$$

The output signal of detector without ellipsoid reflector is

$$
V=R_{0} \sum_{i} I_{0}\left(\lambda_{i}\right) e^{-\alpha\left(\lambda_{i}\right) L_{0}} \frac{S}{L_{0}^{2}}
$$

$\rho(\lambda)$ can be seen as a constant for $\lambda$. 
Let

$$
\begin{aligned}
I_{A}^{\prime} & =\sum_{i} I_{0}\left(\lambda_{A i}\right) e^{-\alpha\left(\lambda_{A i}\right) L_{0}}, \\
I_{A R}^{\prime} & =\sum_{i} I_{0}\left(\lambda_{A i}\right) e^{-\alpha\left(\lambda_{A i}\right) L} \\
I_{B}^{\prime} & =\sum_{i} I_{0}\left(\lambda_{B i}\right) e^{-\alpha\left(\lambda_{B i}\right) L_{0}}
\end{aligned}
$$

In this design, the dual-wavelength differential absorption technology is employed. The signal wavelength $\lambda_{A}$ is $1.665 \mu \mathrm{m}$ from the NIR light source of $5.1 \mathrm{~nm}$ PbSe QDs. The reference wavelength $\lambda_{B}$ is $1.943 \mu \mathrm{m}$ from the NIR light source of $6.1 \mathrm{~nm}$ PbSe QDs. The NIR QDs lights are controlled in a time-sharing way.

Dividing (5) by (6), the differential signal can be obtained as

$$
\begin{aligned}
V_{A B}^{\prime} & =\frac{V_{A}^{\prime}}{V_{B}}=\frac{I_{A}^{\prime}}{I_{B}^{\prime}}+\frac{0.5 \pi \rho L_{0}^{2}}{S} \frac{I_{A R}^{\prime}}{I_{B}^{\prime}}\left[\cos 2 \theta_{1}-\cos 2 \theta_{2}\right] \\
\cdot & {\left[\frac{3}{8}\left(\omega_{1}-\omega_{2}\right)+\frac{1}{4}\left(\sin 2 \omega_{1}-\sin 2 \omega_{2}\right)\right.} \\
+ & \left.\frac{1}{32}\left(\sin 4 \omega_{1}-\sin 4 \omega_{2}\right)\right] .
\end{aligned}
$$

However, the differential signal without ellipsoid is

$$
V_{A B}=\frac{V_{A}}{V_{B}}=\frac{I_{A}^{\prime}}{I_{B}^{\prime}} .
$$

In (8) and (9), $V_{A}$ is the output corresponding to $\lambda_{A}$ without ellipsoid reflector; $V_{A}^{\prime}$ is the output corresponding to $\lambda_{A}$ with ellipsoid reflector; $V_{B}$ is the output corresponding to $\lambda_{B}$, which is almost constant no matter whether there is an ellipsoid reflector.

Therefore, after adding ellipsoidal structure, the gain of the differential signal is

$$
\begin{aligned}
m & =\frac{V_{A B}^{\prime}-V_{A B}}{V_{A B}}=0.5 \pi \rho \frac{L_{0}^{2}}{S} \frac{I_{A R}^{\prime}}{I_{A}^{\prime}}\left[\cos 2 \theta_{1}-\cos 2 \theta_{2}\right] \\
& \cdot\left[\frac{3}{8}\left(\omega_{1}-\omega_{2}\right)+\frac{1}{4}\left(\sin 2 \omega_{1}-\sin 2 \omega_{2}\right)\right. \\
& \left.+\frac{1}{32}\left(\sin 4 \omega_{1}-\sin 4 \omega_{2}\right)\right],
\end{aligned}
$$

where $\Delta L=L-L_{0}$, representing the difference between reflected optical path through ellipsoid and direct incident optical path on the detector.

\section{Experiment and Analysis}

The absorption spectra and PL spectra of the NIR QDs light sources fabricated with $5.1 \mathrm{~nm}$ and $6.1 \mathrm{~nm}$ PbSe QDs are shown in Figures 2 and 3, respectively. The wavelength of $1.66 \mu \mathrm{m}$ is the central wavelength for $\mathrm{CH}_{4}$ detection in the experiment. The near infrared absorption spectrum is shown

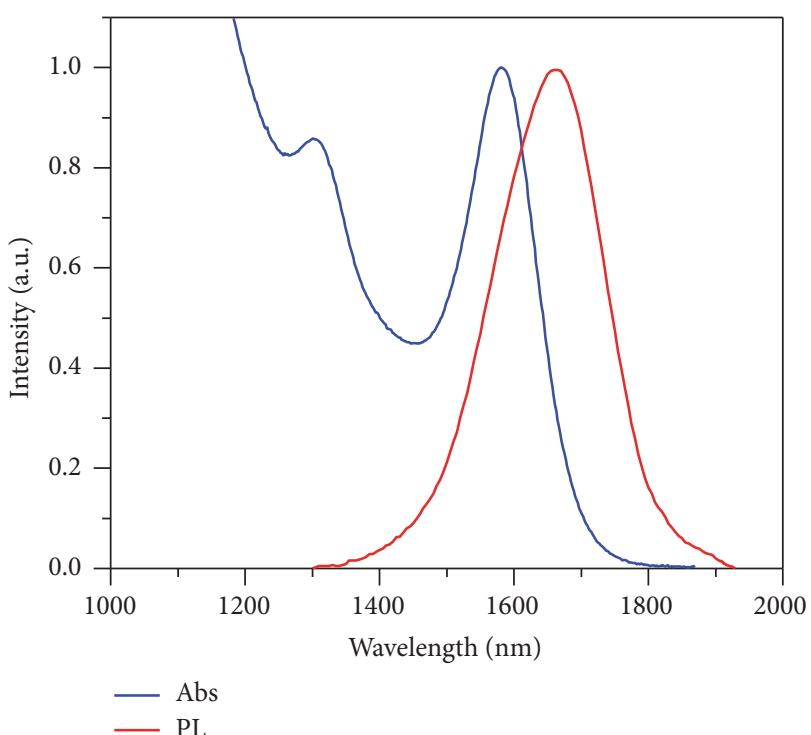

Figure 2: Absorption and PL spectra of $5.1 \mathrm{~nm}$ PbSe QDs.

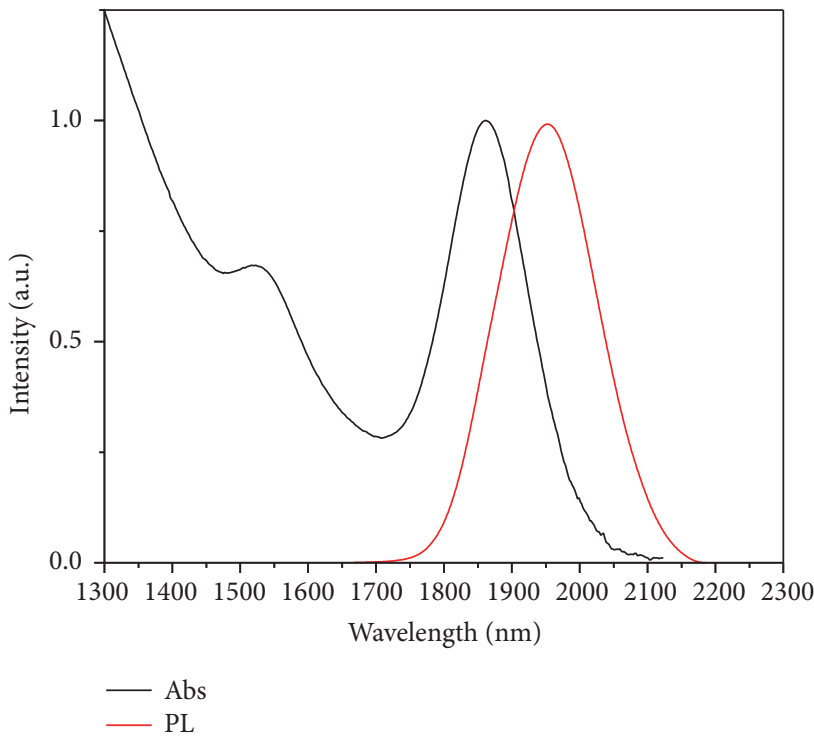

Figure 3: Absorption and PL spectra of $6.1 \mathrm{~nm}$ PbSe QDs.

in Figure 4, which is from HITRAN atmospheric molecular spectroscopy database $[36,37]$.

As seen in Figures 2 and 4, the PL peak of $5.1 \mathrm{~nm}$ PbSe QDs is $1665 \mathrm{~nm}$ with a full width at half-maximum of $143 \mathrm{~nm}$, which covered the entire absorption spectrum of $\mathrm{CH}_{4}$ gas. Carbon dioxide gas, carbon monoxide gas, and water steam are the interference gases in the experiment, whose absorption spectra are shown in Figures 5 and 6. As seen in Figure 5, the absorption coefficients of $\mathrm{CO}$ gas and $\mathrm{CO}_{2}$ gas are small, so the interference influence can be ignored. It can be seen from Figure 6 that the overlap region between the absorption spectrum of $\mathrm{H}_{2} \mathrm{O}$ gas and the PL spectrum of $\mathrm{PbSe} \mathrm{QDs}$ is very small, so the interference effect of water vapor can be considered very small. So, the NIR QDs 


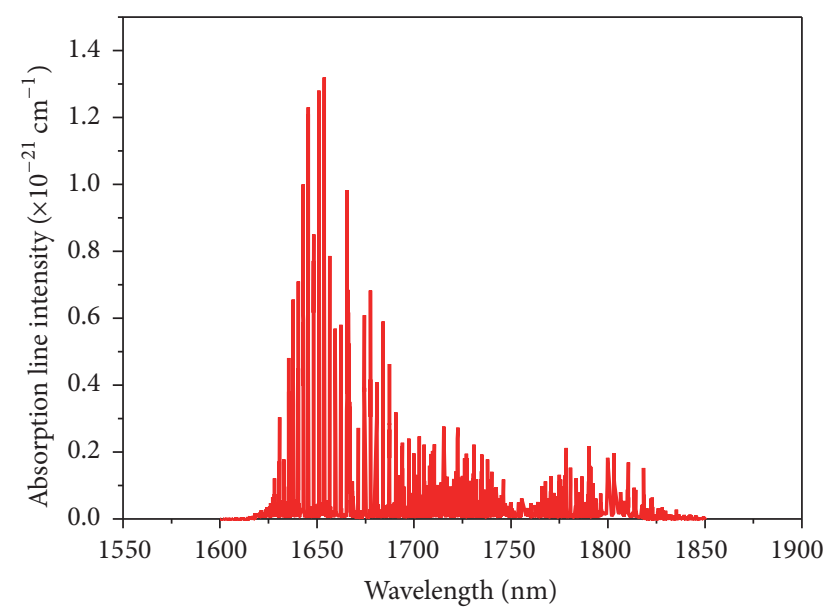

FIgURE 4: Absorption spectra of $\mathrm{CH}_{4}$ near infrared.

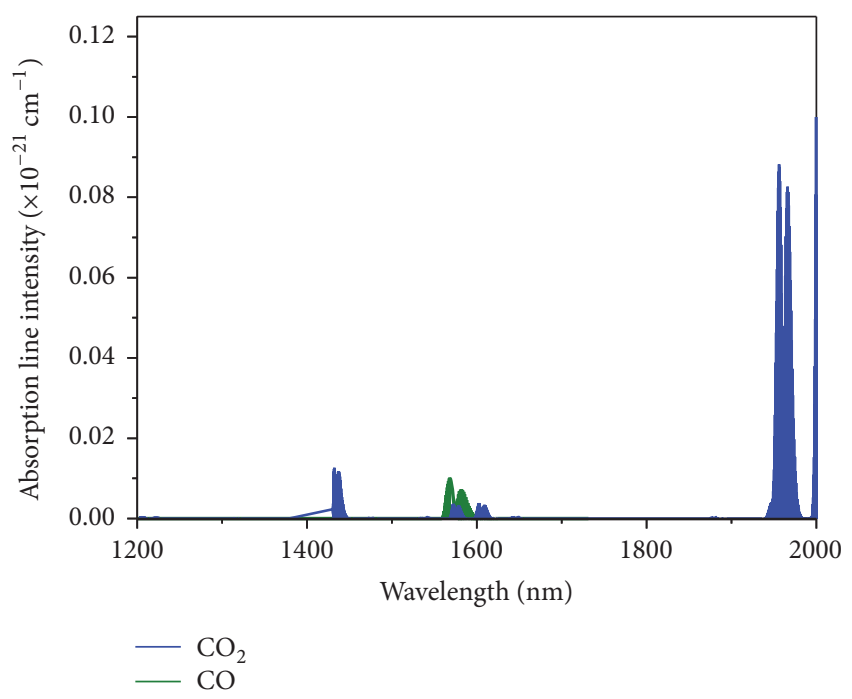

FIgURE 5: Absorption spectra of $\mathrm{CO}$ and $\mathrm{CO}_{2}$ near infrared.

light sources fabricated with $5.1 \mathrm{~nm}$ can be used to detect the concentration of $\mathrm{CH}_{4}$ gas.

Figure 3 indicates the PL peak of $6.1 \mathrm{~nm}$ PbSe QDs is located on $1943 \mathrm{~nm}$ with a full width at half-maximum of $185 \mathrm{~nm}$. As seen in Figures 2 and 3, the two wavelengths are adjacent.

3.1. Differential Signal Gains. Refer to [30, 32]; in the simulation computation, the related parameters are set as follows: $x=0.36 \mathrm{~cm}, \rho=0.95, S=2 \mathrm{~mm} \times 2 \mathrm{~mm}$, and $C=100 \mathrm{ppm}$, where $x$ is the open length of ellipsoid reflector, $\rho$ is the reflectivity of ellipsoid reflector, $S$ is the effective reception area of detector, and $\mathrm{C}$ is the concentration of the $\mathrm{CH}_{4}$ gas. In the ideal case, the different emulational curves of $a$ and $m$ with the increase of the semifocus $c$ are shown in Figure 7.

As seen in Figure 7, the gain of signal relies on $a$ and $c$. When $c$ is fixed, there is only one value of $a$ corresponding to the maximum of gain. With the increase of $a, m$ gradually increases up to maximum and then decreases. It is necessary

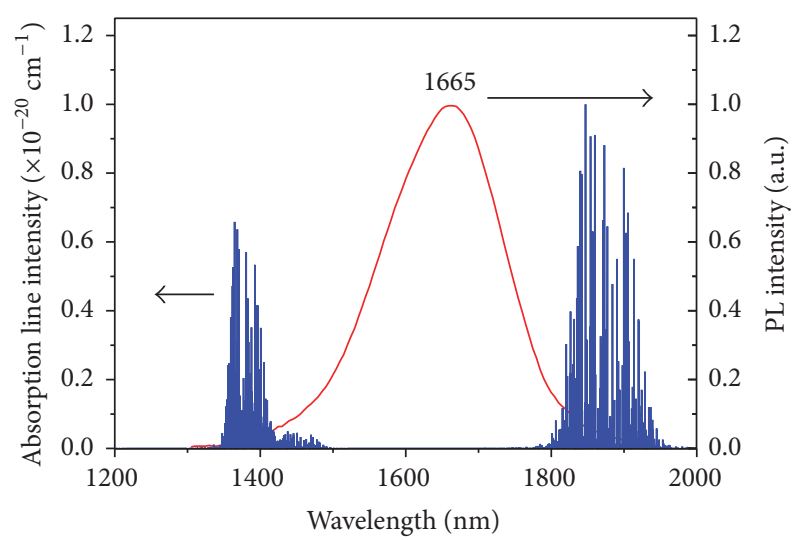

Figure 6: Normalized absorption spectra of PbSe QDs and absorption spectra of $\mathrm{H}_{2} \mathrm{O}$ near infrared.

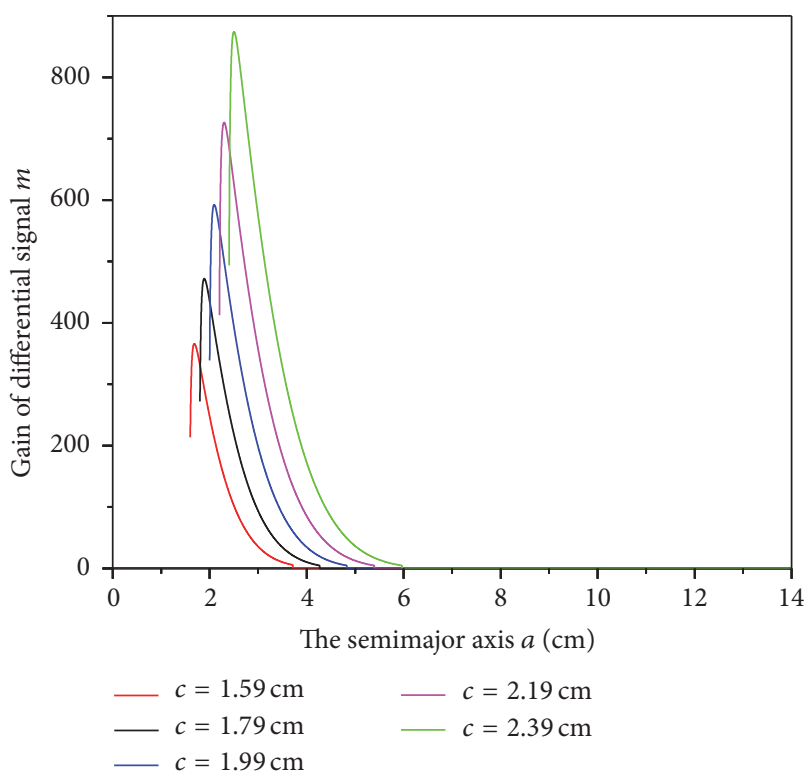

Figure 7: Emulational curves of $a$ and $m$.

to set a reasonable value for $a$ and $c$ of the ellipsoid reflector to get a suitable signal gain. In Figure 7, when we choose $c=$ $1.99 \mathrm{~cm}, a=2.092 \mathrm{~cm}$, and $x=0.36 \mathrm{~cm}$, the signal gain is approximately 593, which is enough to receive a strong signal.

3.2. Surface Curvature Error. Surface curvature of the ellipsoid reflector has manufacture error. We choose $c=1.99 \mathrm{~cm}$ and $x=0.36 \mathrm{~cm}$; the emulational curve of $a$ and $m$ with the fixed manufacture error $\zeta=1^{\circ}$ is shown in Figure 8 .

As shown in Figure 8, when the manufacture error exists, the gain of the differential signal $m$ will be greatly reduced. The maximum decreases from the original 593 to 80 times. This is mainly because that manufacture error will result in the reduction of $\theta_{1}$ and $\theta_{2}$. Further, the reflected lights will not be received by the detector and the reflector surface of ellipsoid cannot be used entirely. 


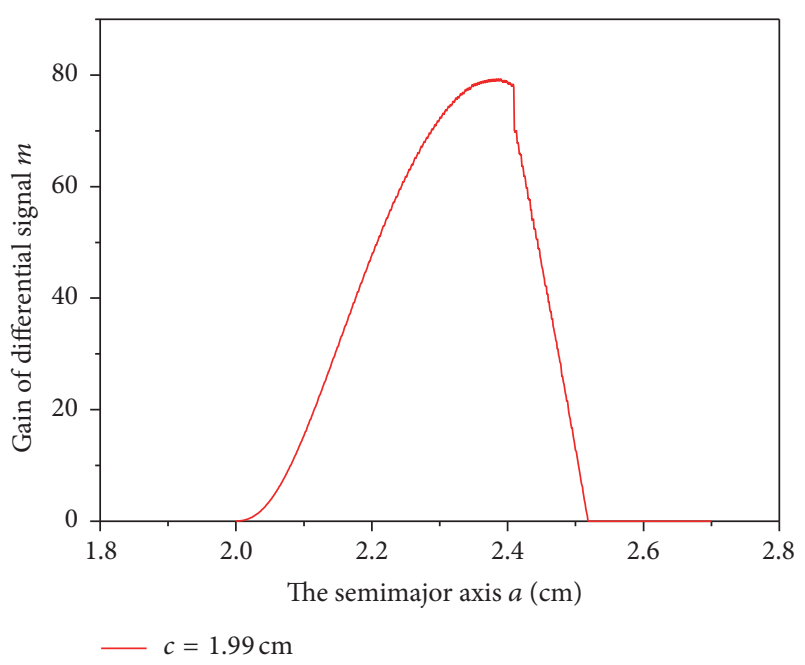

FIGURE 8: Emulational curve of $a$ and $m\left(\zeta=1^{\circ}\right)$.

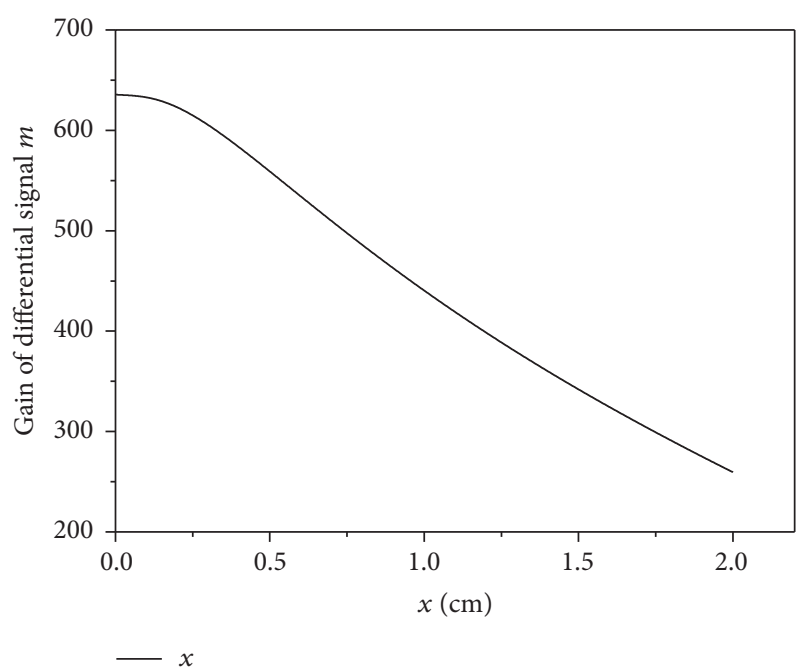

FIGURE 9: Emulational curve of $x$ and $m$.

The output power depends on the differential signal gain $m$. The noisy power of the system is fixed; therefore, the improvement of the signal-to-noise ratio is

$$
\Delta(\mathrm{SNR})_{R}=\frac{\mathrm{SNR}^{\prime}-\mathrm{SNR}}{\mathrm{SNR}}=\frac{P_{A B}^{\prime}-P_{A B}}{P_{A B}}
$$

and $P_{A B}^{\prime} \propto V_{A B}^{\prime 2}, P_{A B} \propto V_{A B}^{2}$; therefore, $\Delta \mathrm{SNR} \propto m^{2}$. In the system, $m \propto 10^{1}$, so the SNR was improved $10^{2}$.

3.3. Open Length. As shown in (10), the open length of the ellipsoid reflector $x$ will lead to some changes of $\theta$ and $\omega$, which will bring some impacts on the signal gain $m$. After $a$ and $c$ are fixed, we simulate the relationship between gain $m$ and $x$ in Figure 9, where $c=1.99 \mathrm{~cm}$ and $a=2.092 \mathrm{~cm}$.

As seen in Figure 9, when $a$ and $c$ are fixed, the gain of the differential signal $m$ is monotonous index decreasing with the increasing of $x$.

\section{Conclusion}

In this paper, the NIR light sources fabricated with PbSe QDs and a new gas cell structure using an ellipsoid reflector were designed to test the concentration of $\mathrm{CH}_{4}$. Based on the double wavelengths differential detection method, the math model of the ellipsoid reflector is established. And the relationship between the parameters of the reflector and the light intensity of the detector is described. It can be proved that the structure can enhance the intensity of the output signal. In simulation, based on the chosen parameters, the differential gain signal could be enhanced 80 times and the SNR could be improved $10^{2}$. In conclusion, the ellipsoid reflector structure will be convenient for the signal amplifying, $\mathrm{AD}$ converting, and other process in the latter circuits, which can be used to improve the detection sensitivity and precision in gas detection system.

\section{Competing Interests}

The authors declare that they have no competing interests.

\section{Acknowledgments}

This work is supported by Department of Science and Technology Plan Projects of Jilin Province under Grant no. 20140101084JC and the "Chunhui" Project of the National Ministry of Education (no. Z2014138).

\section{References}

[1] Y. Zhang, W. Gao, Z. Song et al., "Design of a novel gas sensor structure based on mid-infrared absorption spectrum," Sensors and Actuators, B: Chemical, vol. 147, no. 1, pp. 5-9, 2010.

[2] L. Yan, Y. Zhang, T. Zhang et al., "Tunable near-infrared luminescence of PbSe quantum dots for multigas analysis," Analytical Chemistry, vol. 86, no. 22, pp. 11312-11318, 2014.

[3] S. Tan, W.-F. Liu, L.-J. Wang et al., "Mid-infrared distributedfeedback quantum cascade laser-based photoacoustic detection of trace methane gas," Spectroscopy and Spectral Analysis, vol. 32, no. 5, pp. 1251-1254, 2012.

[4] H. Qiu, M.-J. Liu, and X.-F. Tian, "Pre-alarming apparatus for earthquake based on mid-infrared trace methane detection," Spectroscopy and Spectral Analysis, vol. 34, no. 6, pp. 1524-1527, 2014.

[5] J. N. Wang, L. J. Zheng, X. T. Niu, C. T. Zheng, Y. D. Wang, and F. K. Tittel, "Mid-infrared absorption-spectroscopy-based carbon dioxide sensor network in greenhouse agriculture: development and deployment," Applied Optics, vol. 55, no. 25, pp. 7029-7036, 2016.

[6] H. Wang, D. Cen, G. Men, and J. Wang, "An infrared absorption gas detection system applying plane blazed grating," Advanced Materials Research, vol. 233-235, pp. 2665-2668, 2011.

[7] Z. Jiang, G. You, L. Wang et al., "Solution-processed highperformance colloidal quantum dot tandem photodetectors on flexible substratess," Journal of Applied Physics, vol. 116, no. 8, pp. 084303-084307, 2014.

[8] J. Jing, Y. Zhang, J. Liang et al., "One-step reverse precipitation synthesis of water-dispersible superparamagnetic magnetite 
nanoparticles," Journal of Nanoparticle Research, vol. 14, no. 4, pp. 827-835, 2012.

[9] C. J. Dong, W. X. Yu, M. Xu et al., "Evidence of room temperature ferromagnetism in Co-doped transparent $\mathrm{CuAlO}_{2}$ semiconductor," Journal of Alloys and Compounds, vol. 512, no. 1, pp. 195-198, 2012.

[10] Z. Wang, C. P. Puls, N. E. Staley et al., “Technology ready use of single layer graphene as a transparent electrode for hybrid photovoltaic devices," Physica E: Low-Dimensional Systems and Nanostructures, vol. 44, no. 2, pp. 521-524, 2011.

[11] D. U. Lee, D. H. Kim, D. H. Choi et al., "Microstructural and optical properties of CdSe/CdS/ZnS core-shell-shell quantum dots," Optics Express, vol. 24, no. 2, pp. A350-A357, 2016.

[12] D. Y. Yun, W. S. Song, T. W. Kim, and S. W. Kim, "Electrical stabilities and carrier transport mechanisms of flexible organic bistable devices based on CdSe-InP core-shell nanoparticle/polystyrene nanocomposites," Applied Physics Letters, vol. 5, no. 5, pp. 103305-103309, 2012.

[13] D. V. Talapin, J.-S. Lee, M. V. Kovalenko, and E. V. Shevchenko, "Prospects of colloidal nanocrystals for electronic and optoelectronic applications," Chemical Reviews, vol. 110, no. 1, pp. 389458, 2010.

[14] X. Bai, A. Pucci, V. T. Freitas, R. A. S. Ferreira, and N. Pinna, "One-step synthesis and optical properties of benzoate- and biphenolate-capped $\mathrm{ZrO}_{2}$ nanoparticles," Advanced Functional Materials, vol. 22, no. 20, pp. 4275-4283, 2012.

[15] Z. Tan, Y. Zhang, C. Xie et al., "Near-band-edge electroluminescence from heavy-metal-free colloidal quantum dots," Advanced Materials, vol. 23, no. 31, pp. 3553-3558, 2011.

[16] X. Bai, G. Caputo, Z. Hao et al., "Efficient and tuneable photoluminescent boehmite hybrid nanoplates lacking metal activator centres for single-phase white LEDs," Nature Communications, vol. 5, article 5702, 2014.

[17] I. Moreels, B. Fritzinger, J. C. Martins, and Z. Hens, "Surface chemistry of colloidal PbSe nanocrystals," Journal of the American Chemical Society, vol. 130, no. 45, pp. 15081-15086, 2008.

[18] W. Hu, R. Henderson, Y. Zhang et al., "Near-infrared quantum dot light emitting diodes employing electron transport nanocrystals in a layered architecture," Nanotechnology, vol. 23, no. 37, pp. 375202-375208, 2012.

[19] L. Zhang, Y. Zhang, S. V. Kershaw et al., "Colloidal PbSe quantum dot-solution-filled liquid-core optical fiber for $1.55 \mu \mathrm{m}$ telecommunication wavelengths," Nanotechnology, vol. 25, no. 10, Article ID 105704, 2014.

[20] G. Springholz, T. Schwarzl, W. Heiss et al., "Midinfrared surface-emitting PbSe/PbEuTe quantum-dot lasers," Applied Physics Letters, vol. 79, no. 9, pp. 1225-1227, 2001.

[21] A. G. Ardakani, S. M. Mahdavi, and A. R. Bahrampour, "Time-dependent theory for random lasers in the presence of an inhomogeneous broadened gain medium such as PbSe quantum dots," Applied Optics, vol. 52, no. 6, pp. 1317-1324, 2013.

[22] V. Petkov, I. Moreels, Z. Hens, and Y. Ren, "PbSe quantum dots: finite, off-stoichiometric, and structurally distorted," Physical Review B - Condensed Matter and Materials Physics, vol. 81, no. 24, Article ID 241304, 2010.

[23] A. R. Bahrampour, H. Rooholamini, L. Rahim, and A. A. Askari, "An inhomogeneous theoretical model for analysis of PbSe quantum-dot-doped fiber amplifier," Optics Communications, vol. 282, no. 22, pp. 4449-4454, 2009.

[24] R. Li, Z. Ye, W. Kong, H. Wu, X. Lin, and W. Fang, "Controllable synthesis and growth mechanism of dual size distributed $\mathrm{PbSe}$ quantum dots," RSC Advances, vol. 5, no. 3, pp. 1961-1967, 2015.
[25] P. Gu, Y. Zhang, Y. Feng et al., "Real-time and on-chip surface temperature sensing of GaN LED chips using PbSe quantum dots," Nanoscale, vol. 5, no. 21, pp. 10481-10486, 2013.

[26] Y. Zhang, Q. Dai, X. Li, B. Zou, Y. Wang, and W. W. Yu, "Beneficial effect of tributylphosphine to the photoluminescence of PbSe and PbSe/CdSe nanocrystals," Journal of Nanoparticle Research, vol. 13, no. 9, pp. 3721-3729, 2011.

[27] K. R. Choudhury, Y. Sahoo, T. Y. Ohulchanskyy, and P. N. Prasad, "Efficient photoconductive devices at infrared wavelengths using quantum dot-polymer nanocomposites," Applied Physics Letters, vol. 87, no. 7, pp. 073110-073112, 2005.

[28] X. Gong, Z. Yang, G. Walters et al., "Highly efficient quantum dot near-infrared light-emitting diodes," Nature Photonics, vol. 10, no. 4, pp. 253-257, 2016.

[29] G. Zaiats, D. Yanover, R. Vaxenburg, J. Tilchin, A. Sashchiuk, and E. Lifshitz, "PbSe-based colloidal core/shell heterostructures for optoelectronic applications," Materials, vol. 7, no. 11, pp. 7243-7275, 2014.

[30] Y. Zhang, F.-R. Wang, Y.-H. Zhao et al., "Experiment research on ellipsoidal structure methane using the absorption characteristics of $3.31 \mu \mathrm{m}$ mid-infrared spectroscopy," Infrared Physics and Technology, vol. 55, no. 4, pp. 353-356, 2012.

[31] W. L. Ye, Y. D. Wang, and Z. W. Song, "Design and optimization of IR detection gas cell structure," Semiconductor Optoelectronics, vol. 1, no. 31, pp. 144-147, 2010.

[32] G.-L. Li, M. Dong, N. Song, F. Song, C.-T. Zheng, and Y.D. Wang, "Carbon monoxide gas detection system based on mid-infrared spectral absorption technique," Spectroscopy and Spectral Analysis, vol. 34, no. 10, pp. 2839-2844, 2014.

[33] F. Scholkmann, S. Kleiser, A. J. Metz et al., "A review on continuous wave functional near-infrared spectroscopy and imaging instrumentation and methodology," NeuroImage, vol. 85, pp. 6-27, 2014.

[34] K. Gilmore and P. H. Seeberger, "Continuous flow photochemistry," Chemical Record, vol. 14, no. 3, pp. 410-418, 2014.

[35] J. Yang, N. J. Kramer, K. S. Schramke et al., "Broadband absorbing exciton-plasmon metafluids with narrow transparency windows," Nano Letters, vol. 16, no. 2, pp. 1472-1477, 2016.

[36] A.-L. Sahlberg, J. Zhou, M. Aldén, and Z. S. Li, "Non-intrusive in situ detection of methyl chloride in hot gas flows using infrared degenerate four-wave mixing," Journal of Raman Spectroscopy, vol. 46, no. 8, pp. 695-701, 2015.

[37] M. R. Wang and T. D. Cai, "Theoretical and experimental study on line intensities of $\mathrm{CO}_{2}$ and $\mathrm{CO}$ transitions near $1.5 \mathrm{um}$ at hign temperatures," Acta Physica Sinica, vol. 21, no. 64, Article ID 213301, pp. 213301-213312, 2015. 

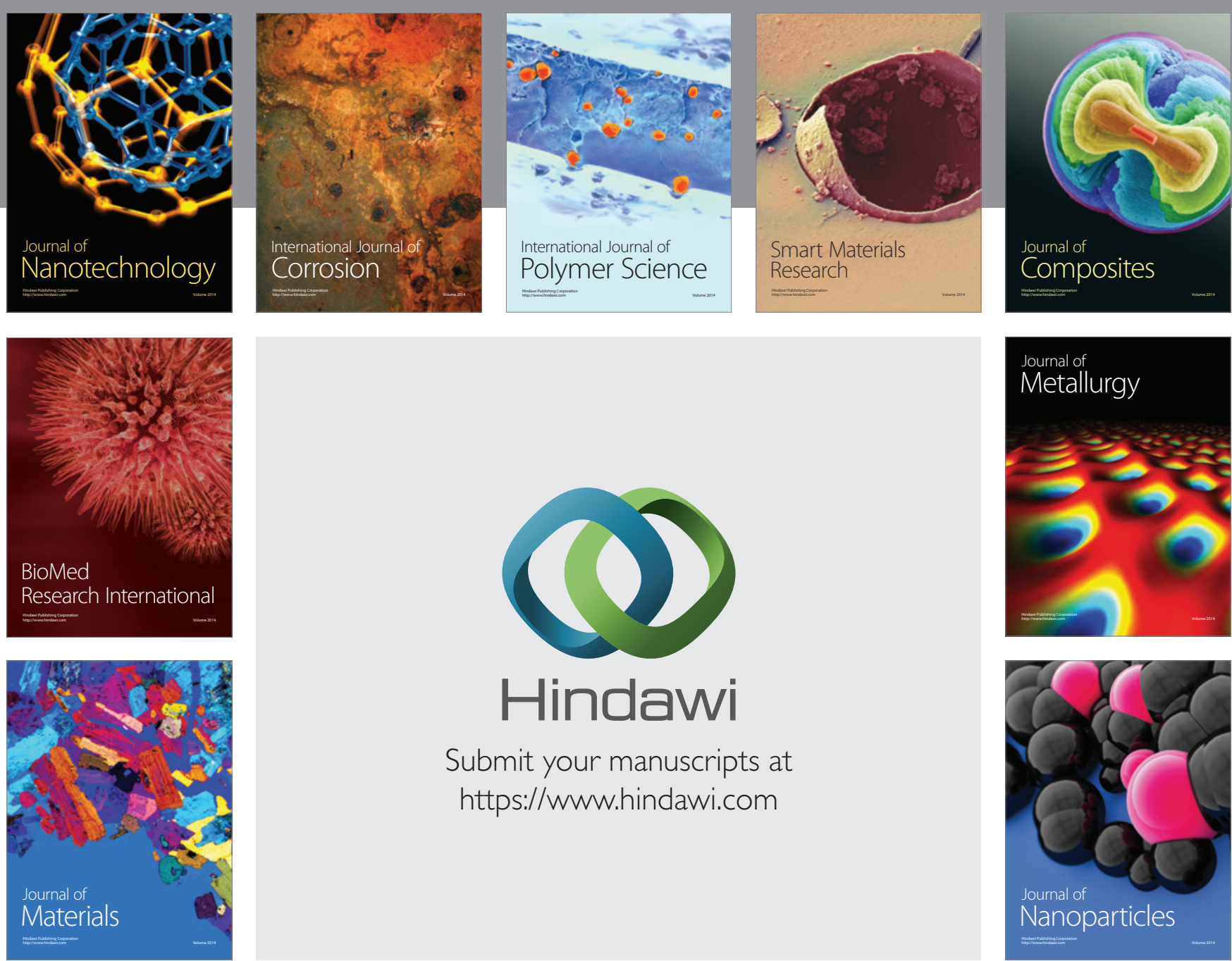

\section{Hindawi}

Submit your manuscripts at

https://www.hindawi.com

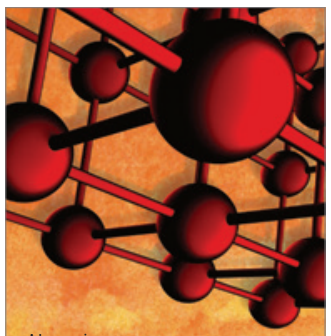

Materials Science and Engineering
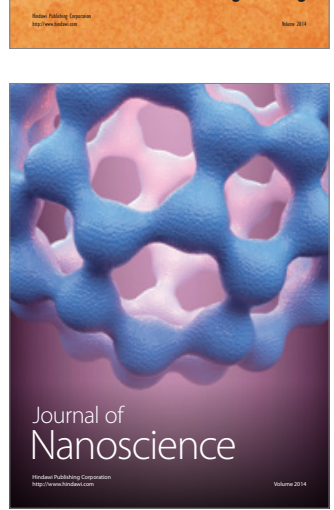
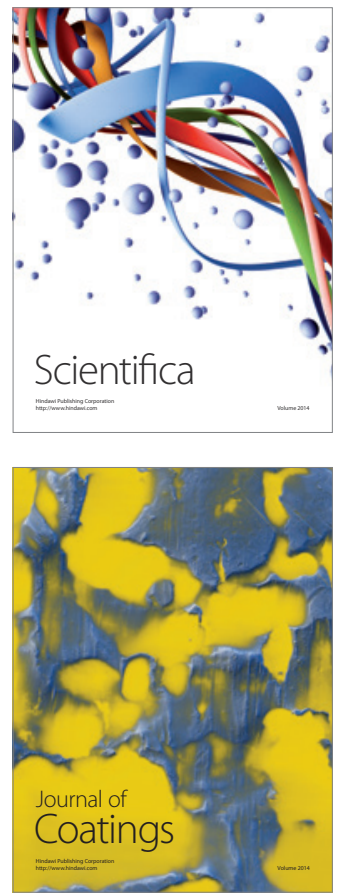
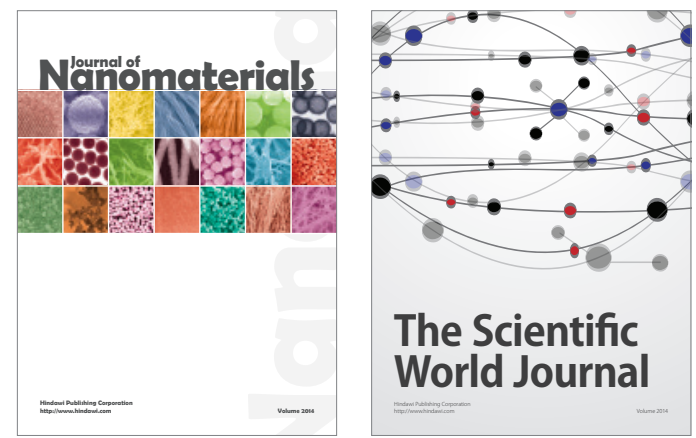

The Scientific World Journal
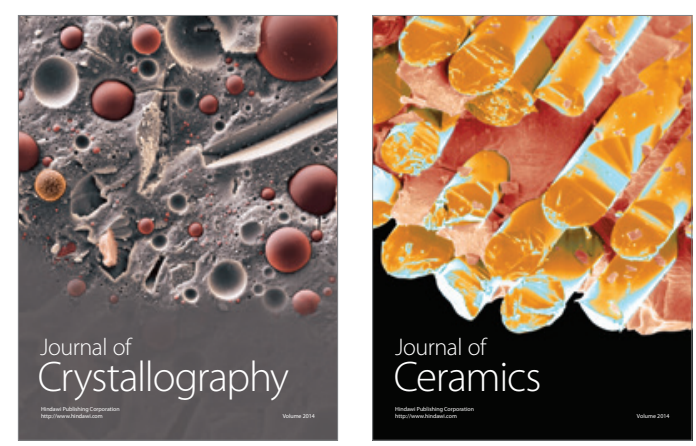
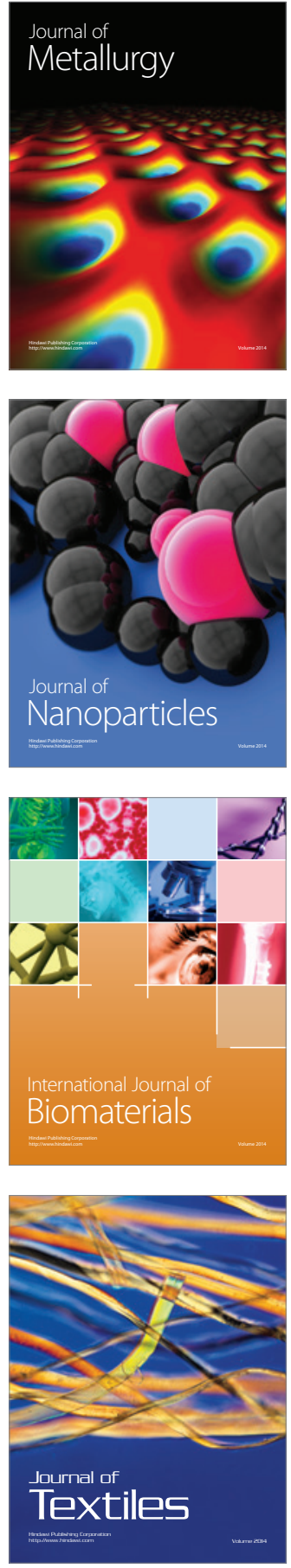